\title{
Determination of the deformation potentials of $\mathrm{GaAs}_{0.80} \mathrm{P}_{0.20}$
}

\author{
Y. González, G. Armelles, and L. González \\ Centro Nacional de Microelectrónica-CSIC, Serrano 144, 28006 Madrid, Spain
}

(Received 28 February 1994; accepted for publication 18 April 1994)

\begin{abstract}
Deformation potentials can be determined by measuring the variation of the energy of the electronic transitions with strain. In this work, the hydrostatic and shear potentials of the band-gap electronic transition $\left(E_{0}\right)$ and the transitions along the $\langle 111\rangle$ direction $\left(E_{1}\right)$ of $\mathrm{GaAs}_{1-x} \mathrm{P}_{x}, x \approx 0.20$, have been determined by electroreflectance characterization of $\mathrm{GaAs}_{1-x} \mathrm{P}_{x}$ layers with different levels of strain.
\end{abstract}

The knowledge of the modification of the physical properties of III-V ternary compounds as a function of the composition is a very important subject, mainly due to its great technological importance. However, the variation of some magnitudes with alloy composition still remains unknown. ${ }^{1,2}$ This is the case of the deformation potentials, magnitudes which describe the effect of the strain on the electronic transitions.

In this communication we present experimental results obtained with electroreflectance measurements, which allow for the determination of the deformation potentials of the $E_{1}$ and $E_{0}$ transitions for $\mathrm{GaAs}_{1-x} \mathrm{P}_{x}$ layers with a phosphorus content about $20 \%$. As we will show later in this work, the deformation potentials values can be determined by measuring the variation of the energy of the electronic transitions with strain.

In this work a different level of strain in the $\mathrm{GaAs}_{1-x} \mathrm{P}_{x}$ $(x \approx 0.20)$ layers is obtained by growing layers of constant composition and different thickness on GaAs substrates (lattice mismatch to GaAs, $f \approx-7.2 \times 10^{-3}$ ). As lattice mismatch relaxation depends on layer thickness, the residual strain in the $\mathrm{GaAs}_{1-x} \mathrm{P}_{x}$ layers will change from one layer to another, allowing for the determination of the deformation potentials for $\mathrm{GaAs}_{0.80} \mathrm{P}_{0,20}$ alloys.

The samples studied in this work consisted of a series of different thickness $h(h=60,200,400 \mathrm{~nm}) \mathrm{GaAs}_{1-x} \mathrm{P}_{x}$ layers of $\mathrm{P}$ composition nearly constant $(0.2 \leqslant x \leqslant 0.24)$ grown on semi-insulating GaAs (001) substrates by atomic layer molecular beam epitaxy (ALMBE). ${ }^{3}$ Growth substrate temperature $T_{s}$ was kept at $350^{\circ} \mathrm{C}$ and special group $V$ element solid sources with fast acting valves and cracking sections were used. Prior to $\mathrm{GaAs}_{1-x} \mathrm{P}_{x}$ layer growth, a $100 \mathrm{~nm}$ thick GaAs buffer layer was grown by conventional molecular beam epitaxy (MBE) at $T_{s}=580^{\circ} \mathrm{C}$.

The experimental procedure which allows for the growth of $\mathrm{GaAs}_{1-x} \mathrm{P}_{x}$ layers with an accurate control of composition is based on the combination of ALMBE growth and in situ refiectivity difference $(\mathrm{RD})$ characterization technique and has been previously reported. ${ }^{4}$ Basically, the experimental procedure developed consists of analyzing the change in the RD signal which experiments on a GaAs (001) surface with different $G$ a surface coverages when a $P_{2}$ pulse impinges on it. As the amplitude of the RD signal, under our experimental conditions, can be directly related to the Ga surface coverage, ${ }^{5}$ the difference between the RD amplitude before and after a $\mathrm{P}_{2}$ pulse provides the amount of $\mathrm{GaP}$ which has been formed, and so far the phosphorus composition of $\mathrm{GaAs}_{1-x} \mathrm{P}_{x}$ layer.

This procedure allows us to grow $\mathrm{GaAs}_{1-x} \mathrm{P}_{x}$ layers with the desired phosphorus composition by an appropriate design of the opening time and duration of the pulses of the phosphorus cell.

All samples were characterized by double crystal $x$-ray diffraction (DCXRD) in the (+-) Bragg arrangement for the (004) reflection and in the $\theta+\phi, \theta-\phi$ for the (115) reflections. These four rocking curves were taken in the [110] and [110] directions in each sample. From the recorded data and by using a dynamical simulation program, we can obtain both the $\mathrm{P}$ composition, always in coincidence with the in situ determined composition, and the residual strain state in the $\mathrm{GaAs}_{1-x} \mathrm{P}_{x}$ layers.

The energy position of the transitions was obtained by means of electroreflectance measurements. For that purpose a semitransparent gold Schottky barrier was evaporated in the samples and the measurements were made at $80 \mathrm{~K}$. Besides the modulated field, a continuous voltage was applied to the sample. Its magnitude was varied in order to record the spectra in the low field regime. ${ }^{6}$ To eliminate the small linear electro-optical (LEO) component ${ }^{7}$ present in the spectra of the electronic transition along the $\langle 111\rangle$ direction $\left(E_{1}\right)$, the spectra were recorded with the light polarized parallel to the [100] direction.

The effect of a biaxial strain in the (001) plane on the fundamental transition at the $\Gamma$ point of the band structure $\left(E_{0}\right)$ can be described by means of two deformation potentials ${ }^{8}$ labeled: a (hydrostatic) and $b$ (shear). Such biaxial strain produces a splitting of the $E_{0}$ transition into a light holelike transition (lh) and a heavy hole-like transition (hh). The energy separation between these two transitions is given by $2 b\left(1+2 C_{12} / C_{11}\right) \epsilon$, where $\epsilon$ is the in-plane strain and $C_{i j}$ the elastic moduli. Strain also induces an energy shift on the center of gravity of the two, hh and $\mathrm{lh}$, transitions being its magnitude $2 a\left(1-C_{12} / C_{11}\right) \epsilon$. If the strain is tensilc (as it is in this work) the lh transition is located at lower energy, whereas for a compressive strain the hh transition appears at lower energy.

For the $E_{1}$ and $E_{1}+\Delta_{1}$ transitions, as they occur between states along the $\langle 111\rangle$ directions, a biaxial strain of the present characteristics does not produce any splitting but a modification of the energy separation between $E_{1}$ and $E_{1}+\Delta_{1}$ given by $\left[\Delta_{1}^{2}+(8 / 3) D_{3}^{3}\left(1+C_{12} / C_{11}\right)^{2} \epsilon^{2}\right]^{1 / 2}$, where $\Delta_{1}$ is the spin-orbit splitting and $D_{3}^{3}$ the intraband deforma- 


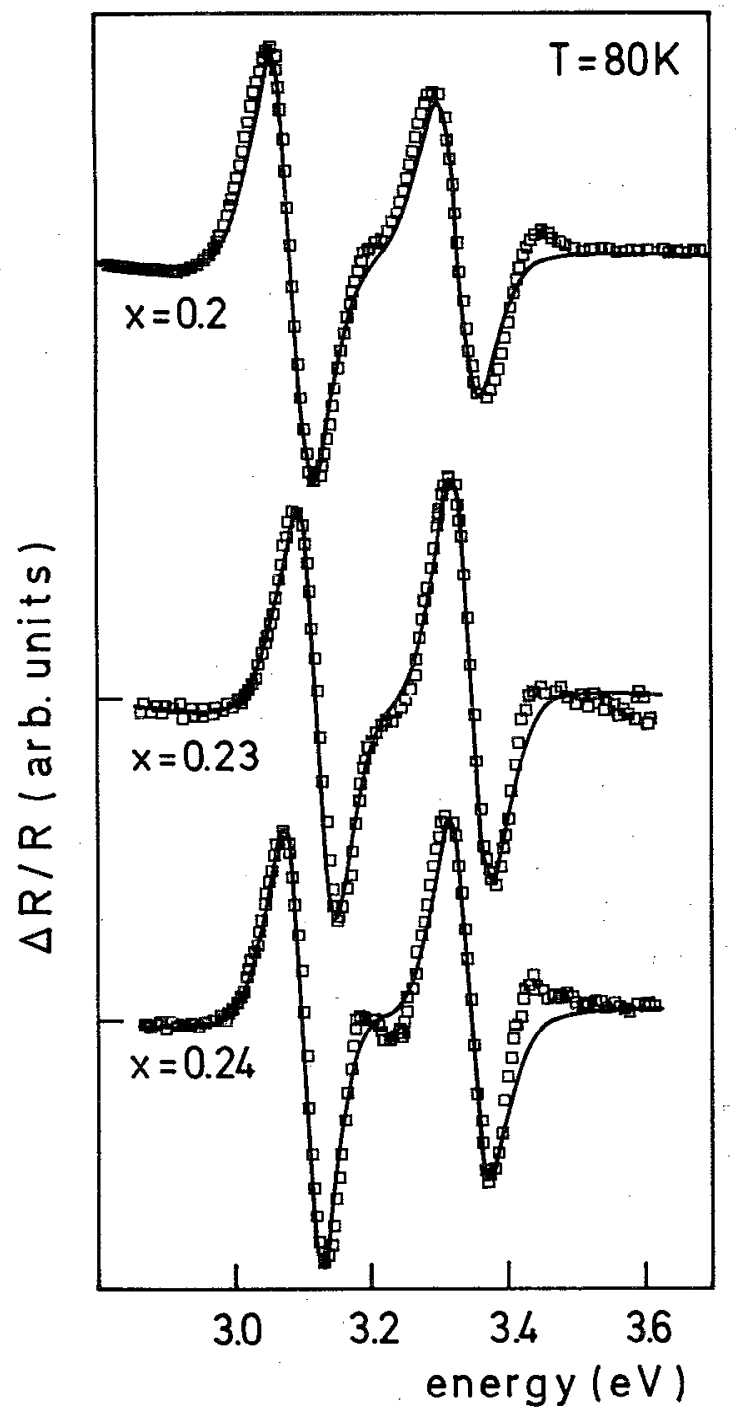

FIG. 1. Electroreflectance spectra of the $\mathrm{GaAs}_{1-x} \mathrm{P}_{x}$ samples studied in this work obtained at $T=80 \mathrm{~K}$ in the $E_{1}$ region. The points are the experimental data and the full curves a theoretical fit using third derivative functional forms. The phosphorus composition determined by double crystal $\mathrm{x}$-ray diffraction are shown in each spectra.

tion potential for the $\Lambda_{3}$ valence band under [001] uniaxial strain. There is also a shift of the energy of the center of gravity of the two transitions given by $2 \epsilon_{1}\left(1-C_{12} / C_{11}\right) \epsilon$ where $\epsilon_{1}$ is the hydrostatic interband $\left(\Lambda_{3}-\Lambda_{1}\right)$ deformation potential.

If the strain field is known the measurement of the splitting between $h \mathrm{~h}$ and $\mathrm{lh}$ transitions directly gives the shear deformation potential $b$, whereas the determination of $D_{3}^{3}$ requires the knowledge of the spin-orbit splitting. The determination of the hydrostatic deformation potentials $a$ and $\epsilon_{1}$, requires the knowledge of the energy of the $E_{0}$ and $E_{1}$ transitions at zero strain, respectively.

In Fig. 1 we show the electroreflectance spectra obtained in the $E_{1}$ region and in Fig. 2 those obtained in the $E_{0}$ region. The points are the experimental data and the full curves a theoretical fit using third derivatives functional forms. ${ }^{6}$ As expected, the strain produces a splitting of the

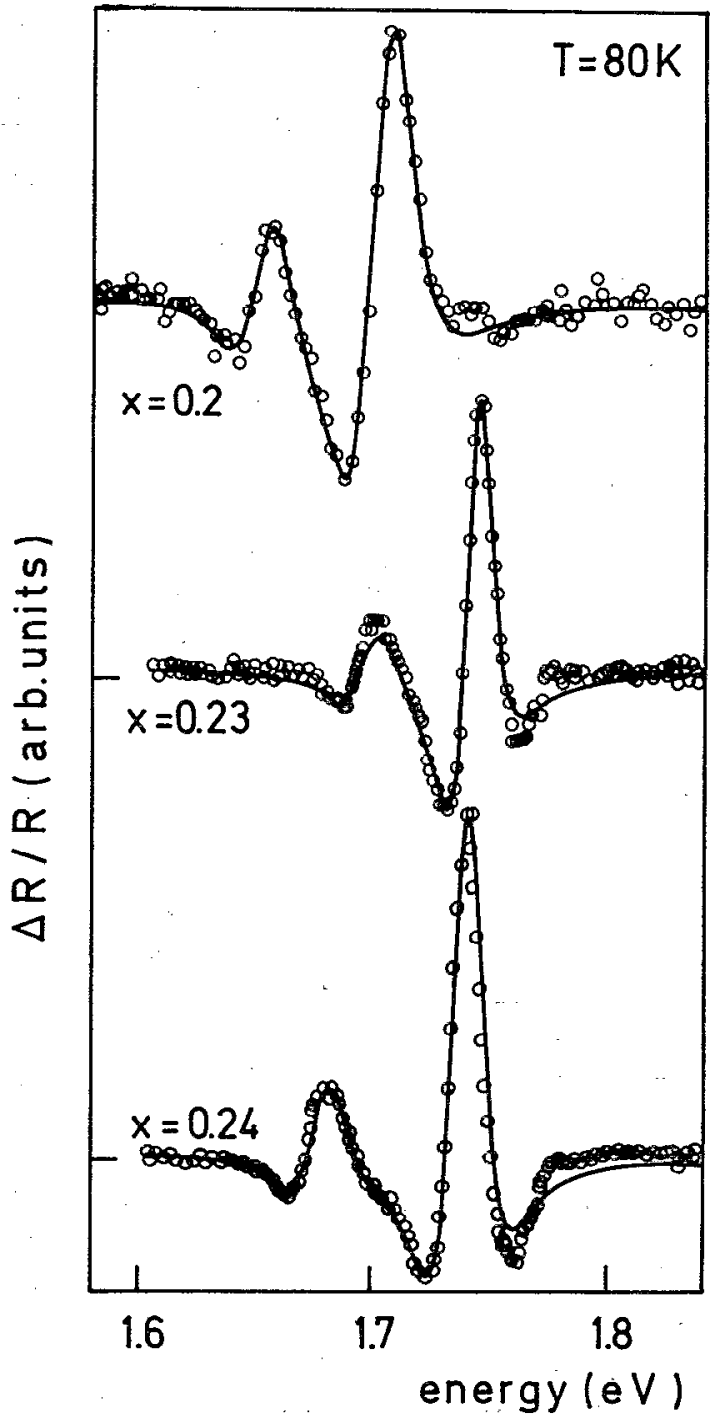

FIG. 2. Electroreflectance spectra of the GaAs $s_{1-x} \mathrm{P}_{x}$ samples studied in this work obtained at $T=80 \mathrm{~K}$ in the $E_{0}$ region. The points are the experimental data, and the full curves a theoretical fit using third derivative functional forms. The phosphorus composition determined by double crystal $x$-ray diffraction are shown in each spectra.

fundamental transition into lh-like and hh-like transitions. In our experimental configuration, with the incident and reflected light polarized in the $(001)$ plane, the intensity of the lh-like transition should be three times lower than the intensity of the hh-like transition, as it is observed. The energies obtained with these fits are given in Table I.

Once we have experimentally determined the modification of the $E_{0}$ and $E_{1}$ transitions dependence on strain, previously obtained by DCXRD characterization, we can directly obtained the deformation potentials values by using the above referred expressions. The energy of the $E_{0}$ and $E_{1}$ transitions at zero strain have been taken from Ref. 9. The values of the deformation potentials obtained in this way are shown in Table I. In Table II we show the deformation potential of the binary compounds. ${ }^{10}$ Due to the strong dispersion in the experimental values of the deformation potentials of the binary compounds, it is difficult to establish any evo- 
TABLE I. Double crystal $x$-ray diffraction determined compositions $(x)$ and residual strain $(\epsilon)$ as well as the thickness $(d)$ of the $\mathrm{GaAs}_{1-x} \mathrm{P}_{x}$ samples studied in this work are shown in this table. $E_{0} \mathrm{hh}, E_{0} \mathrm{hh}, E_{1}$, and $E_{1}+\Delta_{1}$ are the energies of the optical transitions obtained from electroreflectance spectra at $T=80 \mathrm{~K}$ by using a theoretical fit with third derivative functional forms and the obtained values of the deformation potentials.

\begin{tabular}{lccc}
\hline \multicolumn{1}{c}{$x$} & 0.2 & 0.24 & 0.23 \\
\hline$d(\mathrm{~nm})$ & 60 & 200 & 400 \\
$\epsilon\left(\times 10^{3}\right)$ & 7.1 & 8.3 & 6.25 \\
$E_{0} \operatorname{lh}(\mathrm{eV})$ & 1.652 & 1.68 & 1.70 \\
$E_{0} \mathrm{hh}(\mathrm{eV})$ & 1.701 & 1.74 & 1.743 \\
$a(\mathrm{eV})$ & -5.4 & -6 & -5 \\
$b(\mathrm{eV})$ & -1.83 & -1.9 & -1.84 \\
$E_{1}(\mathrm{eV})$ & 3.086 & 3.105 & 3.12 \\
$E_{1}+\Delta_{1}(\mathrm{eV})$ & 3.324 & 3.338 & 3.34 \\
$E_{1}(\mathrm{eV})$ & -4 & -4.5 & -3.4 \\
$D_{3}^{3}(\mathrm{eV})$ & 4.9 & 4.2 & 3.79 \\
\hline \hline
\end{tabular}

lution behavior of the deformation potentials with composition.

In summary we have studied the electroreflectance spectra of a series of $\mathrm{GaAs}_{1-x} \mathrm{P}_{x}$ samples grown by ALMBE on GaAs substrates, with an approximately constant $P$ composition $(0.20 \leqslant x \leqslant 0.24)$ and different layer thicknesses. Due to the lattice mismatch relaxation dependence on layer thickness, the residual strain in the $\mathrm{GaAs}_{1-x} \mathrm{P}_{x}$ layers changes from one sample to another, and this fact allows us to determine the hydrostatic and shear deformation potentials of the $E_{0}$ and $E_{1}$ transitions of $\mathrm{GaAs}_{1}{ }_{x} \mathrm{P}_{x}$ for a composition $x \approx 0.20$.

The authors wish to thank the CEC under ESPRIT II
TABLE II. Values of the deformation potentials of the binary compounds constituents of the $\mathrm{GaAs}_{1-x} \mathrm{P}_{x}$ alloy. Data of this table, unless specified, are taken from Landolt-Börnstein, New Series, Group III, Numerical Data and Functional Relationships in Science and Technology, edited by $O$. Madelung (Springer, Berlin, 1982), Vol. 17, Pt. a.

\begin{tabular}{|c|c|c|c|c|}
\hline \multirow[b]{2}{*}{$a(\mathrm{eV})$} & \multicolumn{2}{|c|}{ GaAs } & \multicolumn{2}{|c|}{$\mathrm{GaP}$} \\
\hline & -6.7 & -9.7 & -9.3 & -9.9 \\
\hline$b(\mathrm{cV})$ & -1.7 & -2 & 1.5 & -1.8 \\
\hline$\in(\mathrm{eV})$ & $-4.3^{\mathrm{a}}$ & -5.4 & \multicolumn{2}{|c|}{-4.3} \\
\hline$D_{3}^{3}(\mathrm{eV})$ & $3.45^{\mathrm{a}}$ & $5.4^{\mathrm{b}}$ & & \\
\hline
\end{tabular}

a Reference 11.

${ }^{\mathrm{b}}$ Reference 12 .

Project 6854 "BLES" (Buffer Layer Engineering in Semiconductors) and the Spanish CICYT under Project No. MAT92-0262 for their support.

${ }^{1}$ S. Adachi, J. Appl. Phys. 53, 8775 (1982).

${ }^{2}$ S. Adachi, J. Appl. Phys. 58, R1 (1985).

${ }^{3}$ F. Briones, L. Gonzalez, and A. Ruiz, Appl. Phys. A 49, 729 (1989).

${ }^{4}$ Y. González, L. González, and F. Briones, J. Cryst. Growth 127, 116 (1993).

${ }^{5}$ F. Briones and Y. Horikoshi; Jpn. J. Appl. Phys. 29, 116 (1990).

${ }^{6}$ D. E. Aspnes, Surf. Sci. 37, 418 (1973).

${ }^{7}$ D. S. Kyser and V. Rehn, Solid State Commun. 8, 1437 (1970).

${ }^{8}$ F. H. Pollak, Semiconductors and Semimetals, edited by T. P. Pearsall (Academic, New York, 1990), Vol. 32, p. 17.

${ }^{9}$ A. G. Thompson, M. Cardona, K. L. Shaklee, and J. C. Woolley, Phys. Rev. 146, 601 (1966).

${ }^{10}$ Landolt-Börnstein, New Series, Group III, Numerical Data and Functional Relationships in Science and Technology, edited by $\mathrm{O}$. Madelung (Springer, Berlin, 1982), Vol. 17, Pt. a.

${ }^{11}$ M. Chandrasekar and F. Pollak, Phys. Rev. B 15, 217 (1977).

${ }^{12}$ P. Etchegoin, J. Kircher, M. Cardona, C. Grein, and E. Bustarret, Phys. Rev. B 46, 15139 (1992). 